\title{
ORIGINAL
}

\section{Variation in severity-adjusted resource use and outcome in intensive care units}

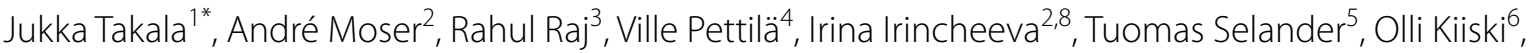 \\ Tero Varpula ${ }^{4}$, Matti Reinikainen ${ }^{7}$ and Stephan M. Jakob ${ }^{1}$ (D)
}

() 2021 The Author(s)

\begin{abstract}
Purpose: Intensive care patients have increased risk of death and their care is expensive. We investigated whether risk-adjusted mortality and resources used to achieve survivors change over time and if their variation is associated with variables related to intensive care unit (ICU) organization and structure.
\end{abstract}

Methods: Data of 207,131 patients treated in 2008-2017 in 21 ICUs in Finland, Estonia and Switzerland were extracted from a benchmarking database. Resource use was measured using ICU length of stay, daily Therapeutic Intervention Scoring System Scores (TISS) and purchasing power parity-adjusted direct costs (2015-2017; 17 ICUs). The ratio of observed to severity-adjusted expected resource use (standardized resource use ratio; SRUR) was calculated. The number of expected survivors and the ratio of observed to expected mortality (standardized mortality ratio; SMR) was based on a mortality prediction model covering 2015-2017. Fourteen a priori variables reflecting structure and organization were used as explanatory variables for SRURs in multivariable models.

Results: SMR decreased over time, whereas SRUR remained unchanged, except for decreased TISS-based SRUR. Direct costs of one ICU day, TISS score and ICU admission varied between ICUs 2.5-5-fold. Differences between individual ICUs in both SRUR and SMR were up to $>3$-fold, and their evolution was highly variable, without clear association between SRUR and SMR. High patient turnover was consistently associated with low SRUR but not with SMR.

Conclusion: The wide and independent variation in both SMR and SRUR suggests that they should be used together to compare the performance of different ICUs or an individual ICU over time.

Keywords: Intensive care unit, Hospital mortality, Health resources, Resource allocation, Health care benchmarking, Cost control

*Correspondence: jukka.takala@med.unibe.ch

${ }^{1}$ Department of Intensive Care Medicine, Bern University Hospital, University of Bern, Bern, Switzerland

Full author information is available at the end of the article

Matti Reinikainen and Stephan M. Jakob shared senior authorship with equal contribution.

\section{Introduction}

The primary goal of intensive care is to prevent death and disability. Intensive care requires extensive resources. In the United States, for example, intensive care costs represent $0.7 \%$ of the gross national product [1]. Since severity of illness influences the risk of death, severity scoring systems have been created for comparison of mortality outcomes [2-7]. Standardized mortality ratio (SMR; the ratio between observed and predicted, severity-adjusted mortality) is widely used to compare the performance of individual intensive care units (ICUs). Several-fold differences in mortality, adjusted for severity of illness, have 
been reported between ICUs both internationally and within countries [6, 8-10]. Much less is known about resources needed to produce survivors of critical illness. Using data from the SAPS-3 database, several-fold differences in severity-adjusted ICU resources needed to produce hospital survivors (standardized resource use ratio, SRUR; SRUR $>1$ indicates more resources than expected used to produce survivors) were observed when ICU length of stay (LOS) was used as a surrogate for resource use [11]. The variation of SRUR was much greater than that of SMR. Such variation may be due to differences in health care systems and economies, or LOS as sole surrogate for resource use [11, 12]. A better understanding of variation in SRUR may help to optimize resource allocation.

The aim of this study was to evaluate variation in SRUR and SMR over time, factors related to this variation, and to analyze cost structures and ICU organization in Finland, Estonia, and Switzerland.

\section{Materials and methods}

We used data from 2008 to 2017, extracted into an anonymized database from a benchmarking database [13]. The study protocol, database contents and data management process were approved by the National Institute of Health and Welfare, Finland (Decision THL/1524/5.05.00/2017 and THL/1173/05.00/2018). According to regulations in Finland, Estonia, and Switzerland, no ethics committee approval was needed.

The Finnish Intensive Care Consortium (FICC) consists of all adult ICUs in Finland except one neurosurgical ICU (19 of 21 ICUs included), and the ICUs of one university hospital in Estonia (100\% of university ICU admissions and $16 \%$ all ICU admissions in Estonia) and one in Switzerland (33\% of all university hospital ICU admissions and $11 \%$ of all ICU admissions in Switzerland). Services with subunits but sharing resources were considered as one ICU (two university hospitals). All ICUs were multidisciplinary. The ICUs were a priori categorized as university hospital ICU (tertiary referral center function), large, or small non-university ICUs (large with primary referral population at least 120,000 inhabitants and at least 6 beds, others small).

Data on diagnosis, severity of illness, care interventions, physiologic, administrative, and hospital outcome data from all ICU admissions are extracted from electronic patient records (manually from paper in Estonia) into a validation software. Using logical rules, median filtering and graphic displays to ensure data quality, each admission is validated by trained ICU nurse datamanagers or intensivists before transfer to the FICC database.

\section{Take-home message}

Severity of illness adjusted hospital mortality in intensive care patients has substantially decreased over time, without an increase in severity-adjusted resources use. The wide and independent variation in both mortality and resource use suggests that both should be used together and adjusted for severity of illness to compare performance of different ICUs or an individual ICU over time.

\section{Study populations}

Overall population included all resources used by the participating ICUs (Fig. 1). For hospital survivor count, readmissions were excluded (SRUR population). Cardiac surgery patients and readmissions were excluded from SMR calculation (SMR population). In both SRUR and SMR populations, admissions missing hospital outcome were excluded. Cost analyses were done for 17 ICUs and years 2015-2017 of the Overall and SRUR populations.

\section{Resource use}

Resource use was assessed using ICU LOS, daily collected Therapeutic Intervention Scoring System-76 (TISS) Scores [14] including 17 additional items (Supplementary Information; referred throughout using prefix "e": eTable1), and direct ICU costs (salaries, drugs, fluids, disposables). The TISS score ranks ICU activities according to intensity and resource use; the sum of daily scores indicates total resource use. First, all units were included using ICU LOS- and TISS-based SRUR for description of changes in SRUR and SMR in 2008-2017. Second, an analysis using direct costs was performed for 2015-2017. ICUs without clear cost allocation were excluded. To include physicians' salaries allocated to other budgets or simultaneously covering other services, physician staff organization, rotations, in-house and on-call coverage was clarified with each ICU leader and costs were allocated based on consensus time estimates (eTable 2).

\section{Calculation of SRUR}

All yearly ICU admissions were stratified according to SAPS-II scores $(0-9,10-19, \ldots, 80-89,>90)$. First, in each stratum, the sums of all LOS days and TISS scores (Overall population) were divided by the number of hospital survivors (SRUR population). This was the expected resource use (as LOS/TISS)/survivor for each stratum. Second, for each ICU, the expected resource use for each SAPS-II stratum was calculated as (number of survivors)"(expected LOS/TISS per survivor) and the expected total resource use as the sum of expected resource use in all SAPS-II strata. Third, the SRUR $\mathrm{LOS}$

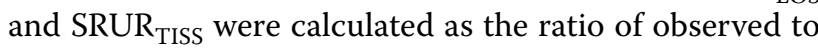




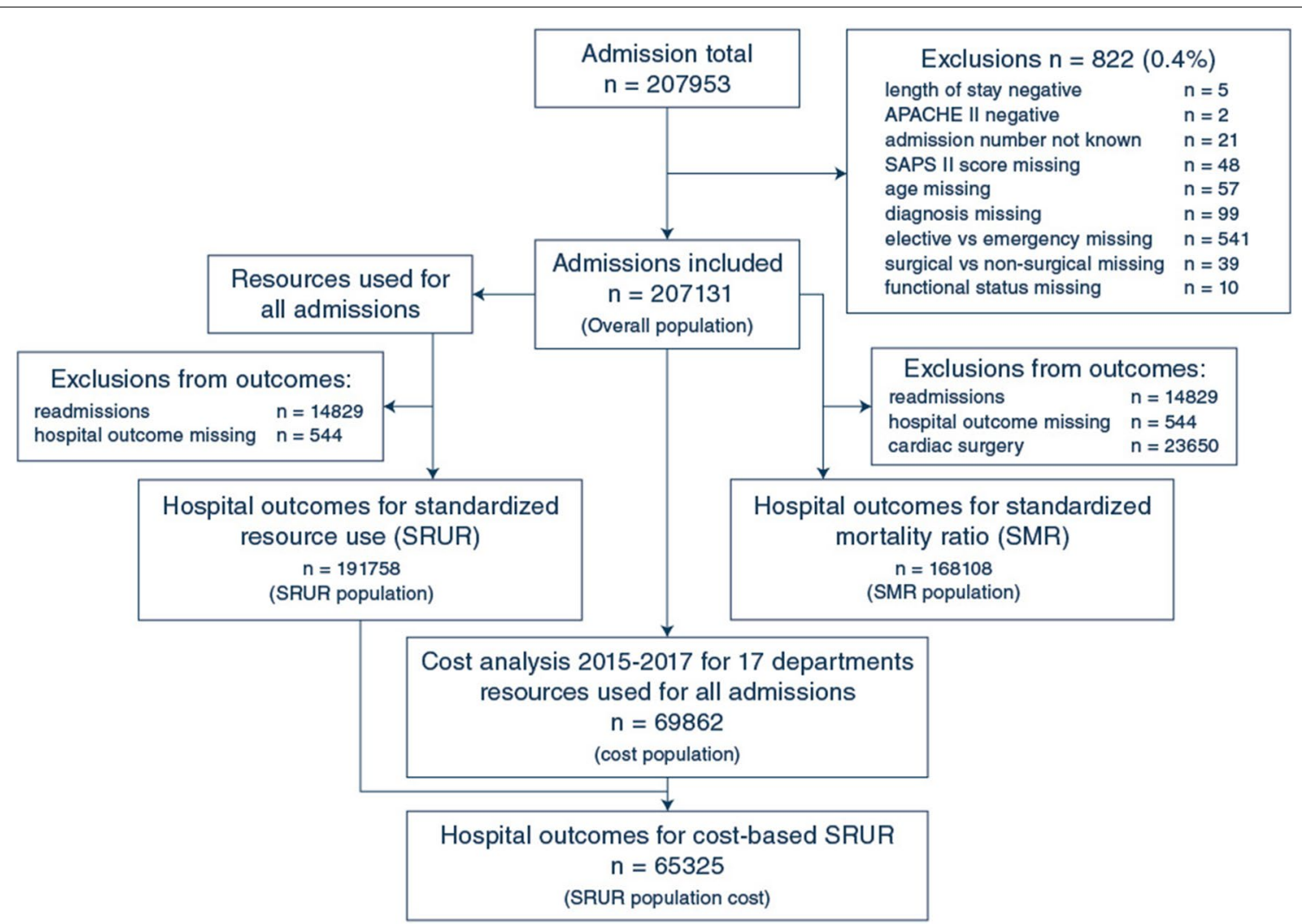

Fig. 1 Flowchart showing study populations and exclusions

expected total resource use, using LOS and TISS, respectively (Supplementary Information: "example of SRUR calculation").

For SRUR using direct costs (years 2015-2017, 17 ICUs), the mean costs of one ICU LOS day and TISS point were calculated as the sum of direct costs for all admissions divided by the sum of LOS days and TISS points, respectively. A fixed exchange rate of 1.00 Swiss franc to 1.09 Euros was used, without inflation adjustment. The expected costs to produce a hospital survivor in each SAPS-II stratum were calculated as (expected resource use LOS/TISS per survivor)*(mean cost of LOS/ TISS). For each ICU, the sum of expected direct costs in all strata (number of survivors"expected costs to produce a hospital survivor) was the expected total direct cost. The cost-based SRUR for each ICU was calculated as observed/expected total direct costs based on LOS (cost-

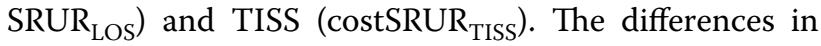
costs between countries were adjusted using the purchasing power parity (PPP) provided by the OECD [15], using $\mathrm{PPP}=1.0$ for Finland (Supplementary Information: "cost adjustment using purchasing power parity"). All costs are PPP-adjusted.

\section{Structure and process}

Each ICU leader provided the average number of beds, physicians (including rotations, in-house and on-call coverage), and nursing staff, organization type (independent/part of another department), availability of an ICU specialist $24 / 7$, availability of a medical emergency team (MET), and presence of other ICU/intermediate care units in campus.

\section{SMR}

SMR was calculated for each ICU as observed/predicted hospital mortality. The predicted probability of hospital mortality was calculated using a recent prediction model customized for FICC, based upon 61,224 ICU admissions between 2015 and 2017 (https://doi.org/10.31219/ osf.io/rb62v). Briefly, the risk prediction model accounts for age, a modified SAPS-II score (without age, admission type), patient pre-admission functional status, APACHEIII diagnosis and admission type (emergency/elective). The predictive performance of the model in the different cohorts is shown in eTable 3. Generally, the discrimination and calibration abilities were very good. 


\section{Statistical analysis}

We described the study population by frequencies $(n)$, percentages (\%), means, standard deviations (SD), median and percentiles. Differences across ICU categories were tested by an analysis of variance and a chisquared test. We used box plots to describe SRUR and SMR by calendar year. We used Gaussian linear regression models to investigate the effect of SMR on SRUR ${ }_{L O S}$ and SRUR $R_{\text {TISS }}$ and the effect of SRUR $R_{\text {LOS }}$ and SRUR

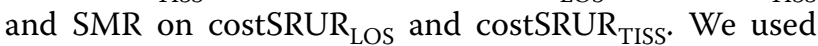
hierarchical regression models to investigate ICU-related factors associated with SRUR $\mathrm{LOS}_{\mathrm{LS}}$, SRUR $\mathrm{TISS}_{\text {, }}$ costSRUR $\mathrm{LOS}_{\mathrm{LO}}$ and costSRUR $\mathrm{TISS}_{\text {and }}$ to investigate an interaction effect between SAPS-strata and ICU category on SRUR and SMR variation for a hypothesized case-mix effect (Supplementary information: "details of regression models"). We a priori selected the following ICU-related variables: ICU category (University, small/large non-University), cardiac surgery in hospital (yes/no), neurosurgery in hospital (yes/no), nurses/bed, nurses/physician, total number of beds, readmissions/total admissions, admissions/bed, specialist availability 24/7 (yes/no), medical emergency team (MET; yes/no), physician full-time equivalents (FTE)/bed (Supplementary Information: "calculation of physician full-time equivalents"), organization type (independent or not), median SAPS-II and SMR. We used bivariable models (one of the above variables adjusted for calendar year) and multivariable models (all of the above variables adjusted for calendar year). ICU category was only used in bivariable models, because of its high correlation with other variables. Continuous variables were standardized (centered and expressed per one standard deviation increase) and relative risk estimates (RR) reported with 95\% CI. Analyses with ICU-category stratification were justified by an interaction (two-sided $p$ value $p<0.05$ ) between SAPS-II and ICU category. Fixed effect estimates for SAPS-II strata and calendar years are expressed as estimated SRUR and SMR changes with 95\% CI. ICU-specific random effects with 95\% CI were used to assess variation across ICUs, by ICU category. All analyses are performed in $\mathrm{R}$ version 4.0.2 ( $\mathrm{R}$ Team Core. $R:$ A language and environment for statistical computing, Vienna, Austria. R Foundation for Statistical Computing).

\section{Results}

\section{ICU and admission characteristics}

From 2008 to 2017, 207,131 admissions were included from 21 units (8 university, 7 large non-university, and 6 small non-university ICUs; Fig. 1). Data for all 10 years were available from 18 hospitals (6 university, 6 large non-university, 6 small non-university ICUs), whereas due to structural or organizational changes, two university ICUs provided data for 2013-2017 and one non-university hospital for 2008-2013 only. Direct costs for 2015-2017 for costSRUR $\mathrm{LOS}_{\text {as }}$ and costSRUR $\mathrm{TISS}_{\text {were }}$ available for 17 ICUs (8 university, 4 large non-university, 5 small non-university ICUs).

The admission characteristics across ICU categories were different (Table 1 ; all $p$ values $<0.001$, except $p=0.02$ for gender). The non-university ICUs treated predominantly emergency $(>90 \%)$ and non-surgical admissions (74\%) with higher median SAPS-II, whereas the university ICUs had more elective (34\%) and surgical admissions (53\%) and the shortest median LOS (key unit characteristics in eTable 2). The university hospitals in the three countries provided $71 \%$ of all ICU admissions, and the two university hospital ICUs in Estonia and Switzerland $45 \%$ of all university hospital admissions.

\section{Analysis of direct costs 2015-2017}

The mean direct costs for one ICU admission, ICU day and TISS point ranged annually from $€ 6487$ to $€ 6774$, from $€ 2082$ to $€ 2125$ and from $€ 51$ to $€ 52$, respectively (eTable 4). The mean direct costs for achieving one hospital survivor were $€ 7183-€ 7500$. The variation between ICUs was high in all cost variables, more than 2.5 -fold in costs of 1 ICU day and one TISS point, and more than 5 -fold in costs of admission and costs for achieving one hospital survivor. The costs/survivor increased exponentially with increasing SAPS-II category $(p<0.001$; eTable 5; eFigure 1), and the variation between ICUs was high. Salary costs contributed $81 \pm 6 \%$ (mean \pm SD) to total direct costs, and the remaining $19 \pm 6 \%$ were costs for drugs, fluids and disposables (eFigures 2, 3).

\section{Evolution of SRUR and SMR over time}

From 2008 to 2017, SMR decreased over time $(p<0.001$; Fig. 2, left panel). There was no overall change over time in median SAPS-II score (range 31-34; eFigure 4). The relative contribution of the highest SAPS strata decreased (calendar year"SAPS stratum interaction $p<0.001$ ). Funnel plots indicated no unexpected SMR variation in relation to number of admissions (eFigure 5).

The resources needed to produce one survivor decreased by $0.7 \%, 95 \% \mathrm{CI}(1.2-0.2 \%)$, when assessed with $\operatorname{SRUR}_{\text {TISS }}(p=0.02$; Fig. 2, left panel), whereas there was no change in $\operatorname{SRUR}_{\mathrm{LOS}}(p=0.27$; Fig. 2, left panel). The costSRUR ${ }_{\text {LOS }}$ and costSRUR ${ }_{\text {TISS }}$ (years 2015-2017) did not change (Fig. 2, right panel). We found an interaction between ICU categories and SAPS strata (all $p<0.001$; SAPS stratified SRUR LOS , SRUR TISS and SMR in each ICU category eFigure 6). Non-university ICUs had wider ranges of SMR and smaller ranges of SRURs (eFigure 7). 
Table 1 Admission characteristics by intensive care unit category and overall

\begin{tabular}{|c|c|c|c|c|}
\hline & $\begin{array}{l}\text { University } \\
(N=147,251)\end{array}$ & $\begin{array}{l}\text { Non-university (large) } \\
(N=36,343)\end{array}$ & $\begin{array}{l}\text { Non-university (small) } \\
(N=23,537)\end{array}$ & $\begin{array}{l}\text { Overall } \\
(N=207,131)\end{array}$ \\
\hline \multicolumn{5}{|l|}{ Age (years) } \\
\hline Mean (SD) & $59.8(17.5)$ & $58.3(19.7)$ & $61(18.6)$ & $59.7(18.1)$ \\
\hline Median [Min, Max] & $63[0,100]$ & $63[0,102]$ & $64[0,101]$ & $63[0,102]$ \\
\hline \multicolumn{5}{|l|}{ Gender } \\
\hline Male & $91,789(62.3 \%)$ & $22,957(63.2 \%)$ & $14,674(62.3 \%)$ & $129,420(62.5 \%)$ \\
\hline Female & $55,435(37.6 \%)$ & $13,386(36.8 \%)$ & $8856(37.6 \%)$ & 77,677 (37.5\%) \\
\hline Missing & $27(0 \%)$ & $0(0 \%)$ & $7(0 \%)$ & $34(0 \%)$ \\
\hline \multicolumn{5}{|l|}{ Type of admission } \\
\hline Readmission & $11,040(7.5 \%)$ & $2402(6.6 \%)$ & 1387 (5.9\%) & $14,829(7.2 \%)$ \\
\hline First admission & $136,211(92.5 \%)$ & $33,941(93.4 \%)$ & $22,150(94.1 \%)$ & $192,302(92.8 \%)$ \\
\hline \multicolumn{5}{|l|}{ Treatment type } \\
\hline Elective & $50,374(34.2 \%)$ & $2184(6 \%)$ & $2109(9 \%)$ & $54,667(26.4 \%)$ \\
\hline Emergency & 96,877 (65.8\%) & $34,159(94 \%)$ & $21,428(91 \%)$ & $152,464(73.6 \%)$ \\
\hline \multicolumn{5}{|l|}{ Surgical treatment } \\
\hline Non-surgical & $69,147(47 \%)$ & $27,692(76.2 \%)$ & $16,701(71 \%)$ & $113,540(54.8 \%)$ \\
\hline Surgical & $78,104(53 \%)$ & $8651(23.8 \%)$ & $6836(29 \%)$ & $93,591(45.2 \%)$ \\
\hline \multicolumn{5}{|l|}{ SAPS-II score stratum } \\
\hline $0-9$ & $6467(4.4 \%)$ & $755(2.1 \%)$ & $517(2.2 \%)$ & 7739 (3.7\%) \\
\hline $10-19$ & 22,904 (15.6\%) & $4199(11.6 \%)$ & $2970(12.6 \%)$ & $30,073(14.5 \%)$ \\
\hline $20-29$ & $41,601(28.3 \%)$ & $8476(23.3 \%)$ & $6127(26 \%)$ & $56,204(27.1 \%)$ \\
\hline $30-39$ & 31,933 (21.7\%) & 8404 (23.1\%) & $5631(23.9 \%)$ & $45,968(22.2 \%)$ \\
\hline $40-49$ & $19,208(13 \%)$ & $6190(17 \%)$ & $3576(15.2 \%)$ & $28,974(14 \%)$ \\
\hline $50-59$ & $12,114(8.2 \%)$ & $3829(10.5 \%)$ & $2175(9.2 \%)$ & $18,118(8.7 \%)$ \\
\hline $60-69$ & 7277 (4.9\%) & $2381(6.6 \%)$ & $1360(5.8 \%)$ & $11,018(5.3 \%)$ \\
\hline $70-79$ & $3352(2.3 \%)$ & $1198(3.3 \%)$ & $680(2.9 \%)$ & $5230(2.5 \%)$ \\
\hline $80-89$ & $1466(1 \%)$ & $540(1.5 \%)$ & $294(1.2 \%)$ & $2300(1.1 \%)$ \\
\hline$\geq 90$ & $929(0.6 \%)$ & $371(1 \%)$ & 207 (0.9\%) & 1507 (0.7\%) \\
\hline \multicolumn{5}{|l|}{ SAPS-II score } \\
\hline Mean (SD) & $33.5(17.2)$ & $37.7(17.9)$ & $36.1(17.4)$ & $34.5(17.5)$ \\
\hline Median [Min, Max] & $30[0,129]$ & $35[0,118]$ & $33[0,135]$ & $31[0,135]$ \\
\hline \multicolumn{5}{|l|}{ TISS EXT score sum } \\
\hline Mean (SD) & $127(212)$ & $128(194)$ & $116(162)$ & $126(204)$ \\
\hline Median [Min, Max] & $62[1,6150]$ & $69[2,4270]$ & $66[2,6120]$ & $64[1,6150]$ \\
\hline Missing & $176(0.1 \%)$ & $13(0 \%)$ & $9(0 \%)$ & $198(0.1 \%)$ \\
\hline \multicolumn{5}{|l|}{ Length of stay (days) } \\
\hline Mean (SD) & $3.05(5.39)$ & $3.28(5.35)$ & $2.91(4.48)$ & $3.07(5.29)$ \\
\hline Median [Min, Max] & $1.1[0,136]$ & $1.6[0.00139,118]$ & $1.6[0.00625,169]$ & $1.22[0,169]$ \\
\hline \multicolumn{5}{|l|}{ Outcome in hospital } \\
\hline Survivor & $132,204(89.8 \%)$ & 30,766 (84.7\%) & $19,997(85 \%)$ & $182,967(88.3 \%)$ \\
\hline Non-survivor & $15,047(10.2 \%)$ & $5577(15.3 \%)$ & $3540(15 \%)$ & $24,164(11.7 \%)$ \\
\hline
\end{tabular}

SAPS-I/ simplified acute physiology score; LOS length of stay in the intensive care unit; TISS Therapeutic Intervention Scoring System; TISS EXT score sum includes the additional items described in the "Methods"

\section{Relationship between SRUR and SMR and between SRUR and costSRUR}

We found no association between the SRUR ${ }_{\mathrm{LOS}}$, SRUR TISS, costSRUR $\mathrm{LOS}_{\mathrm{L}}$ or costSRUR $\mathrm{TISS}_{\text {with }}$ SMR, except for SRUR $_{\mathrm{LOS}}$ from 2008 to 2010 and in 2013 (Fig. 3,
eFigure 8, eTables 6 and 7). An increase in costSRUR $\mathrm{LOS}_{\mathrm{L}}$

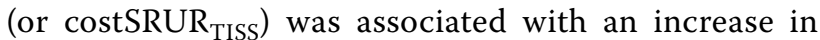

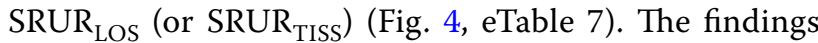
were similar for different ICU categories (eFigures 9, 10). 


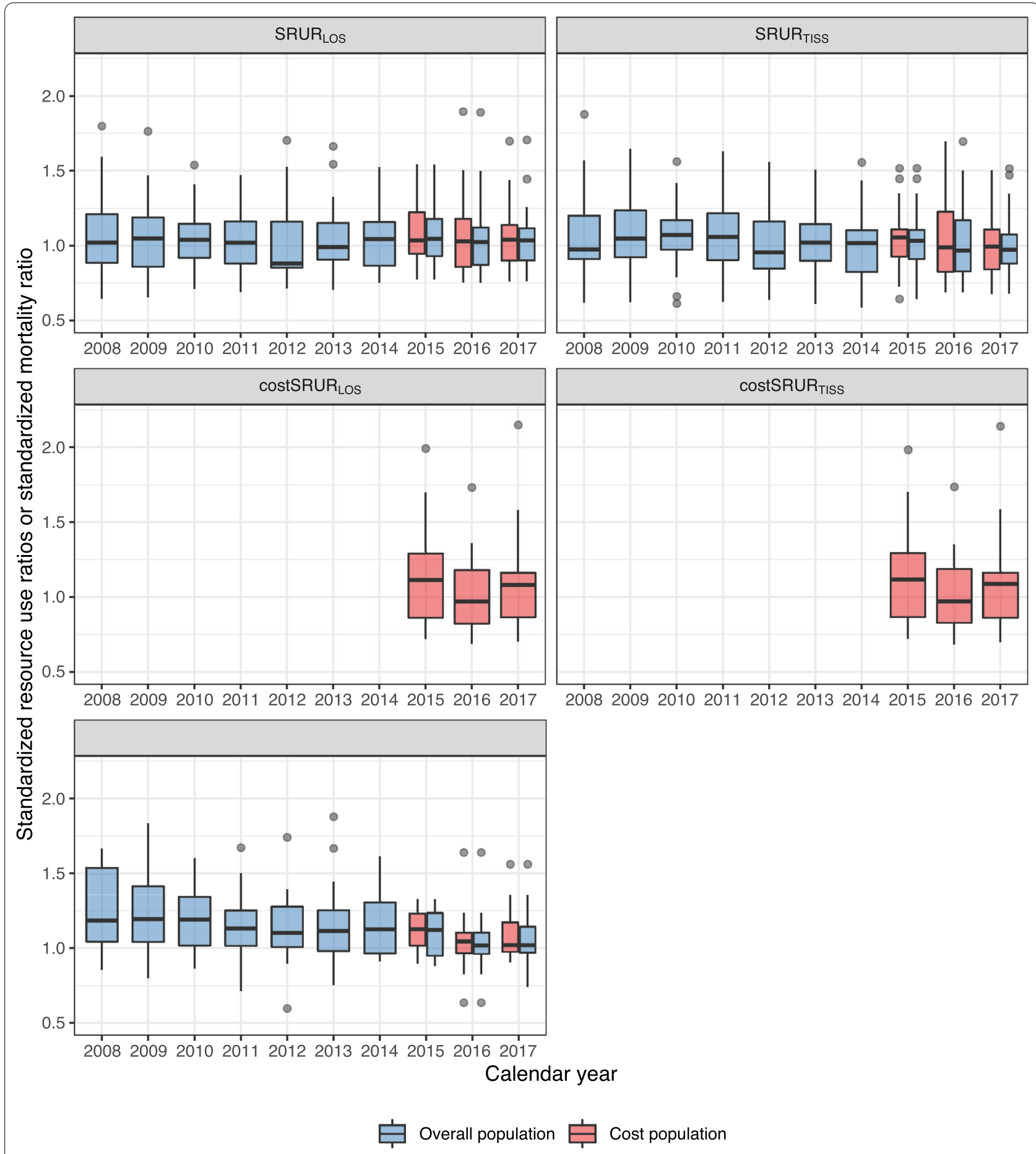

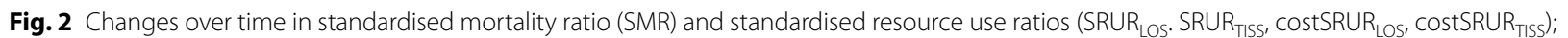
box plots show the median, the first and third quartiles, and whiskers defined by 1.5 times the interquartile range; two-sided $p$ value testing of no

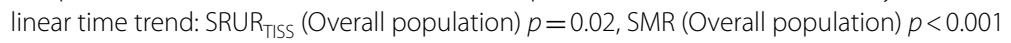

Association of ICU-related factors with SRURs and SMR In multivariable models, the following a priori selected ICU-related variables were significantly associated with decreased SRUR (RR estimates in Fig. 4): higher admissions/bed (all SRURs), independent organization (costSRURs), lower median SAPS-II (costSRURs) and 


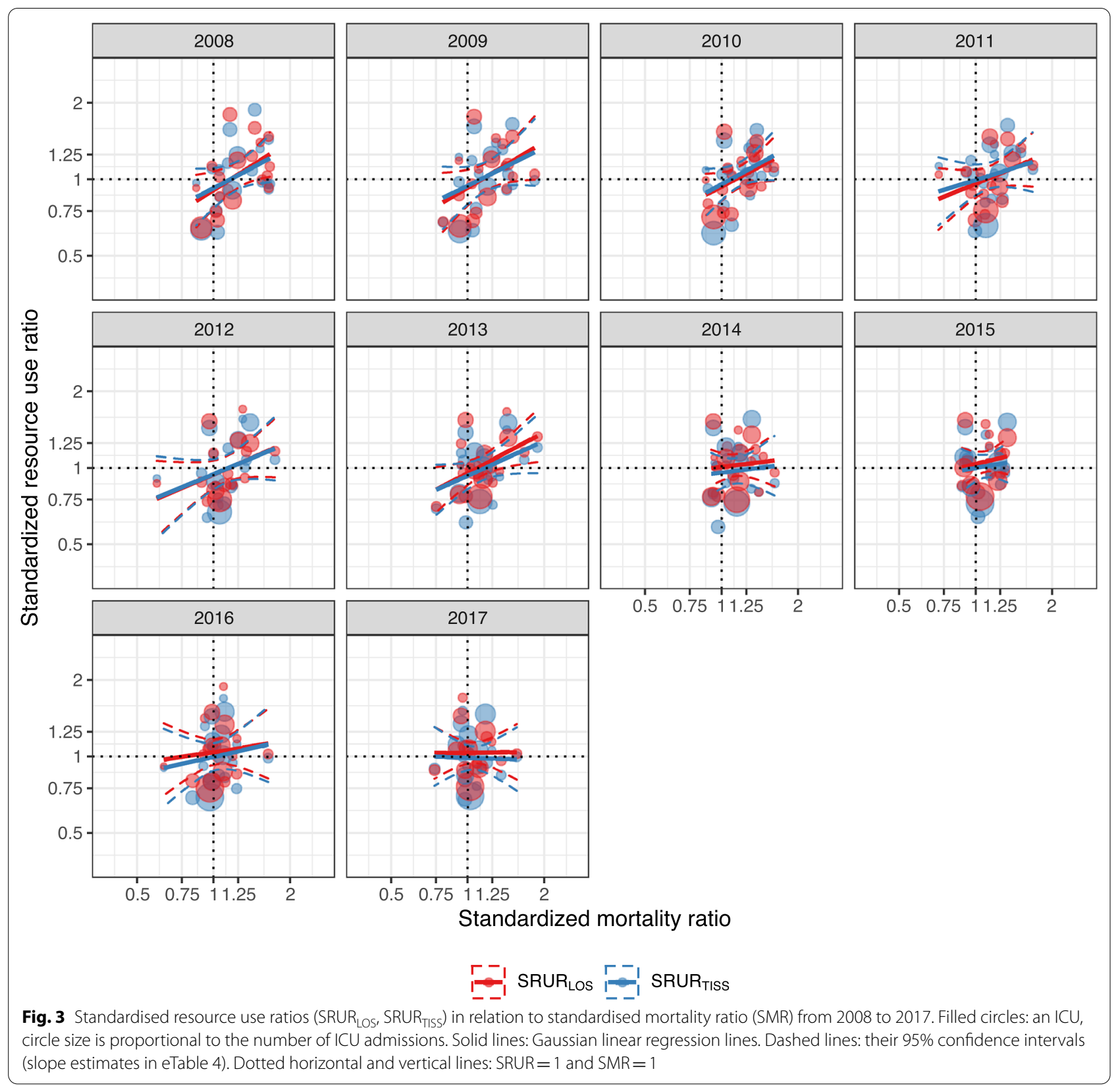

neurosurgery performed in hospital (costSRURs). Variables associated with increased SRUR were: higher SMR $\left(\mathrm{SRU}_{\mathrm{LOS}}, \mathrm{SRUR}_{\mathrm{TISS}}\right.$, costSRUR $\left._{\mathrm{TISS}}\right)$, readmissions/total admissions (all SRURs), nurses/physicians (costSRURs), and nurses/bed (costSRURs), specialist availability 24/7 (costSRURs) and higher FTE physicians/bed (costSRURs) (RR and 95\%CI in eTable 8).

In bivariable models, higher admissions/bed was significantly associated with decreased SRUR (all SRURs), and the following variables were associated with increased SRUR: higher SMR (SRU $\mathrm{LOS}_{\mathrm{LO}}$, $\left.\mathrm{SRUR}_{\mathrm{TISS}}\right)$, and nurses/bed (costSRURs), specialist availability 24/7 $\left(\mathrm{SRUR}_{\mathrm{TISS}}\right)$ and higher FTE physicians/bed (SRUR $\left.\mathrm{TISS}\right)$.

Variables associated with increased SMR were cardiac surgery performed in hospital (only university hospitals), independent organization (multivariable models) and small non-university ICUs (bivariable models), whereas neurosurgery performed in hospital (only university hospitals) was associated with decreased SMR in bivariable models. 


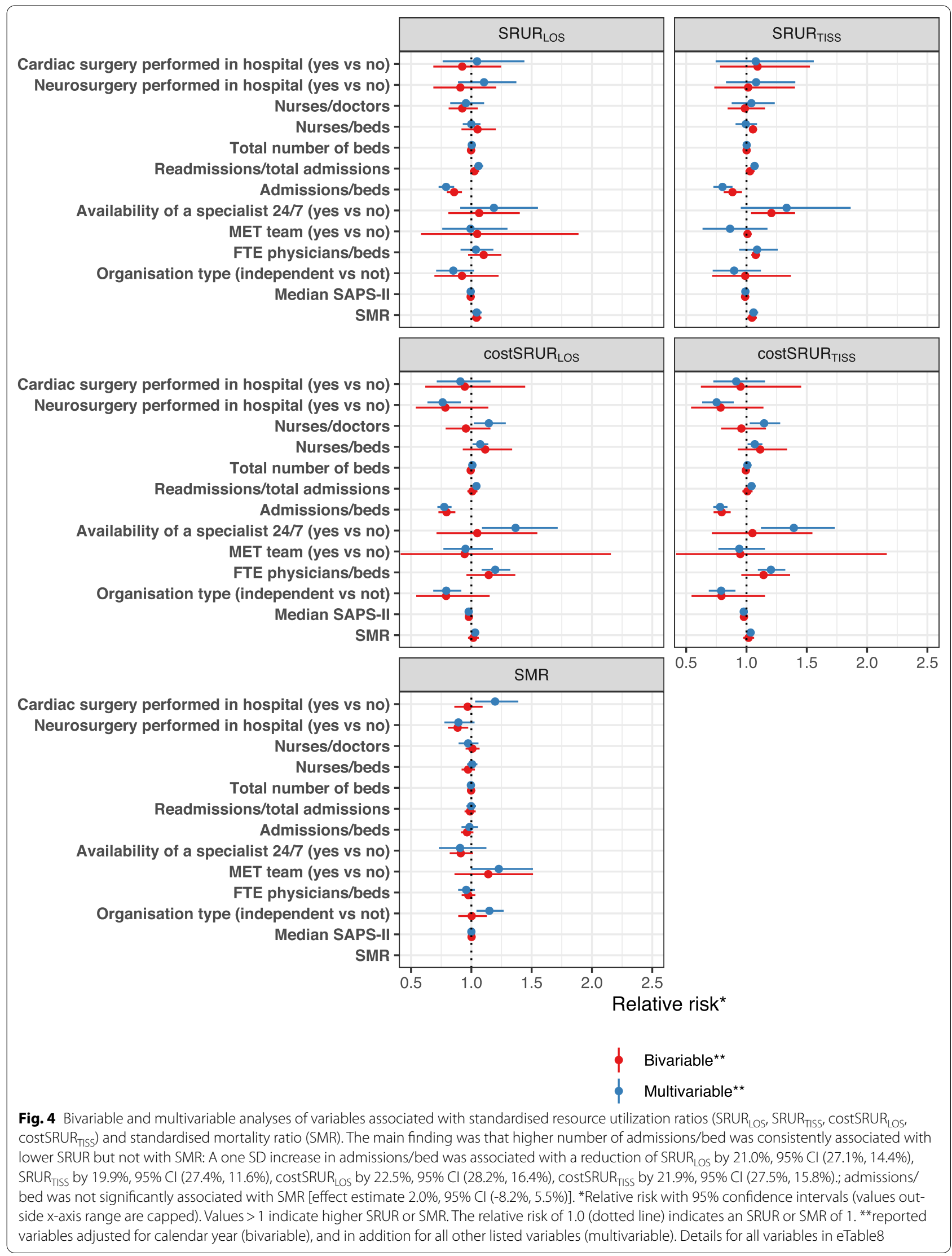




\section{Discussion}

We provide in-depth information on ICU resources and costs needed to achieve hospital survivors in three European countries with different health care systems and wealth over 10 years. We adjusted for case mix between ICUs and for financial strength between countries to enhance comparability.

The main findings were:

(1) Severity-adjusted mortality (SMR) decreased over time, whereas the severity-adjusted resources needed to produce survivors (SRUR)-assessed using the surrogate indicators of LOS and TISS-remained unchanged or decreased.

(2) Differences between individual ICUs in both SRURs and SMR were up to >threefold, their evolution was highly variable, and there was no clear association between SRUR and SMR; nevertheless, the category small non-university ICUs was associated with increased SMR. Furthermore, the university ICUs had higher ranges of SRURs and lower ranges of SMRs than the non-university ICUs.

(3) The direct costs of ICU day, TISS score and ICU admission varied between the ICUs by $2.5-5$-fold. The observed cost ranges agree with previous studies $[16,17]$.

The costs of producing hospital survivors increased exponentially with increased severity of illness and mortality. This makes assessment of resource utilization using any cost components or outcomes meaningless without severity adjustment.

The ICUs' severity-adjusted ability to produce hospital survivors, as reflected by SMR, the resources needed to produce survivors, and the distribution of severity of illness (case mix) are bound to interact. The concept of adjusting resource use for severity of illness was originally proposed by Rothen et al. Using ICU LOS as a surrogate for resource use in 16,560 patients from 275 ICUs from diverse health care systems and socioeconomic conditions, SRUR ${ }_{\text {LOS }}$ was found to vary much more than SMR (highest SRUR $\mathrm{LOS}_{2} \sim 30$ and SMR 3.6) [11]. Soares et al. [12] observed lower variability of SRUR $_{\text {LOS }}$ (highest SRUR LOS $_{2} \sim 6$ ) in 59,693 patients from 78 ICUs in Brazil. The present study on SRUR among ICUs in an established quality consortium is the largest so far, and the first comparing LOS-based and TISS-based SRUR over time, as well as providing direct cost-based data. The variation of both SRUR and SMR was substantially smaller in our study, with SRUR ${ }_{\text {LOS }}$, SRUR $_{\text {TISS }}$ and SMR ranging from $\sim 0.6$ to 1.8 and costSRUR $_{\text {LOS }}$ and costSRUR TISS from $\sim 0.7$ to 2.7 and corresponding SMR of $\sim 0.6$ to 1.5 . This variation is still high, especially since about $2 / 3$ of the admissions were from one country with homogenous health care system, but substantially lower than the variation in direct costs/ admission and direct costs/survivor.

Due to the lack of association between SRURs and SMR, these should be used together to evaluate ICU performance. For example, the wider range of SRURs in the university ICUs versus the wider range of SMRs in the non-university ICUs suggests the need to focus on cost containment in the university ICUs, and on higher quality care in terms of severity-adjusted outcomes in the non-university ICUs.

Low SRUR combined with low SMR should provide the best results of outcome and costs. SMR is widely used to assess ICU performance. With increasing demand to control health care costs without compromising quality, combining SRUR with SMR offers obvious advantages. Severity adjustment of both resource use and outcomes may also be helpful in cost-efficiency studies $[16,18]$. Ideally, resource use indicators should be convertible to costs, but differences in cost accounting, allocation, and budgeting can be major barriers. The surrogate resource use indicators SRUR $_{\mathrm{LOS}}$ and SRUR $\mathrm{TISS}_{\text {correlated well }}$ with the corresponding cost-based SRUR, suggesting that in the absence of sufficient direct costs data, SRUR $\mathrm{LOS}_{\mathrm{L}}$ and SRUR TISS $_{\text {provide comparable information. SRUR as }}$ concept has the potential to be adapted to other health care settings, where indices of illness severity and costs or their surrogates are available. This could facilitate the evaluation of resources needed to care and rehabilitate post-ICU disabilities.

High patient turnover was the main variable associated with low SRUR. This may sound self-evident, but it has not been verified in the context of severity-adjusted costs and outcomes. High turnover with short LOS can be associated with either low severity of illness, high risk of readmissions with poor outcome, or both. We found an association between high turnover and lower SRUR without an association with SMR. That is, a higher number of admissions/bed was not associated with worse outcome but was associated with reduced resource use, both adjusted for severity of illness. Measures to safely increase patient turnover may include changes in clinical practice (e.g. sedation, mobilization, weaning from ventilation, hemodynamic management) and process measures (e.g. daily routines in decision making, bed availability for discharge, enhanced intermediate care capacity).

High patient turnover may also cause strain and lead to worsening outcomes. We did not have the data to assess strain. The annual mean bed occupation rates were always below $80 \%$, and in most ICUs below $60 \%$ (eFigure 11), making strain unlikely. The lack of association 
between number of admissions/bed and SMR suggests that strain, if present, had no major impact on SMR.

Increased nurses/bed and physicians/bed increased the costSRURs without an impact on SMR. In terms of absolute staff numbers, all participating units were well staffed and the nurses/bed (median 0.83 or 1:1.2) exceeds the threshold associated with decreased mortality [19]. The relevance of specialist availability $24 / 7$ is controversial $[19,20]$. Our results suggest that specialist availability $24 / 7$ increases costs but has no consistent impact on SMR.

The association between an independent organization and increased SMR needs to be interpreted with caution. Independent organization was present in five of eight university ICUs and in one large non-university ICU. ICU category was not included in the multivariable model due to its high correlation with other variables. We, therefore, suspect that case-mix differences between ICU categories are likely to contribute.

Many ICU consortia world-wide collect data on SMR and other quality indicators $[7,8,10,12,13]$. SRUR using either surrogates or direct costs if available, can be incorporated relatively easily in such data sets, especially when electronic patient data management systems (PDMS) and medical records are available. We believe that the parallel use of SRUR and SMR can provide relevant information for all intensive care stakeholders.

Our study has several strengths. These include standardized and validated data acquisition with extensive logical controls, use of electronic patient data management systems in all but one center, clear allocation of direct costs, large database with very few missing data, inclusion of $>90 \%$ of the ICU admissions in Finland and of two large university hospitals in countries with different health care systems and wealth, and cost adjustment to PPP. The limitations should also be considered. Only university ICUs from outside Finland were included and they represented 33-100\% university ICU admissions in Switzerland and Estonia, respectively. Their results should not be considered as representative of ICU practices of these countries in general. Although most ICUs had their own budgets, physicians' salaries were often partly allocated to other budgets, due to tasks shared between ICUs and others, especially in the non-university ICUs. We used time-based consensus for the allocation of these costs, using average ICU physician salaries as reference. These potential inaccuracies still represent a small minority of the direct costs and should not cause relevant bias. Thus, we consider our findings as reliable and generalizable for intensive care in high-income countries.

In conclusion, our findings demonstrate wide and independent variation in both SMR and SRUR. SMR and SRUR should, therefore, be used together to compare performance of different ICUs or an individual ICU over time. The parallel use of SMR and SRUR may provide relevant information for all stakeholders involved in intensive care.

\section{Supplementary Information}

The online version contains supplementary material available at https://doi. org/10.1007/s00134-021-06546-4.

\section{Author details \\ ${ }^{1}$ Department of Intensive Care Medicine, Bern University Hospital, Univer- sity of Bern, Bern, Switzerland. ${ }^{2}$ Clinical Trials Unit, University of Bern, Bern, Switzerland. ${ }^{3}$ Department of Neurosurgery, University of Helsinki and Helsinki University Hospital, Helsinki, Finland. ${ }^{4}$ Division of Intensive Care, University of Helsinki and Helsinki University Hospital, Helsinki, Finland. ${ }^{5}$ Science Service Center, Kuopio University Hospital, Kuopio, Finland. ${ }^{6}$ HWE Benchmarking Ser- vices, TietoEvry, Helsinki, Finland. ${ }^{7}$ Department of Anesthesiology and Inten- sive Care, Kuopio University Hospital and University of Eastern Finland, Kuopio, Finland. ${ }^{8}$ Present Address: Biostatistics, CSL Behring, Bern, Switzerland.}

\section{Acknowledgements}

We thank Andreas Limacher for his helpful comments on the statistical analysis and methodology.

\section{Author contributions}

JT: conceptualization, methodology, investigation, resources, data curation, writing —original draft, visualization, supervision, project administration. AM: conceptualization, methodology, software, validation, formal analysis, writing — review and editing, visualization. RR: investigation, resources, data curation, writing - review and editing, supervision, project administration. VP: conceptualization, methodology, writing — review and editing. II: conceptualization, methodology, software, validation, formal analysis, visualization. TS: conceptualization, methodology, writing — review and editing. OK: conceptualization, methodology, writing - review and editing. TV: conceptualization, methodology, writing — review and editing. MR: conceptualization, methodology, writing — review and editing, supervision. SMJ: conceptualization, methodology, resources, writing — review and editing, supervision.

\section{Funding}

Open Access funding provided by Universität Bern. The study was supported by departmental funds of the Department of Intensive Care Medicine, Bern University Hospital, University of Bern, Bern, Switzerland. There was no external funding for this project. Dr. Raj has received research grants from Finska Läkaresällskapet, Svenska Kulturfonden and Medicinska Understödsföreningen Liv \& Hälsa.

\section{Availability of data statement}

The authors had permission from FINDATA (Social and Health Data Permit Authority) to analyse the data. A secondary use of the data for other researchers can be obtained through FINDATA (https://findata.fi/en/) according to the Finish Secondary Data Act.

\section{Declarations}

\section{Conflicts of interest}

On behalf of all authors, the corresponding author states that there is no conflict of interest.

\section{Open Access}

This article is licensed under a Creative Commons Attribution-NonCommercial 4.0 International License, which permits any non-commercial use, sharing, adaptation, distribution and reproduction in any medium or format, as long as you give appropriate credit to the original author(s) and the source, provide a link to the Creative Commons licence, and indicate if changes were made. The images or other third party material in this article are included in the article's Creative Commons licence, unless indicated otherwise in a credit line to the material. If material is not included in the article's Creative Commons licence and your intended use is not permitted by statutory regulation or exceeds the 
permitted use, you will need to obtain permission directly from the copyright holder. To view a copy of this licence, visit http://creativecommons.org/licen ses/by-nc/4.0/.

\section{Publisher's Note}

Springer Nature remains neutral with regard to jurisdictional claims in published maps and institutional affiliations.

Received: 16 July 2021 Accepted: 25 September 2021

Published online: 18 October 2021

\section{References}

1. Halpern NA, Pastores SM (2010) Critical care medicine in the United States 2000-2005: an analysis of bed numbers, occupancy rates, payer mix, and costs. Crit Care Med 38:65-71. https://doi.org/10.1097/CCM. Ob013e3181b090d0

2. Knaus WA, Draper EA, Wagner DP, Zimmerman JE (1985) APACHE II: A severity of disease classification system. Crit Care Med 13:818-829. https://doi.org/10.1097/00003465-198603000-00013

3. Knaus WA, Wagner DP, Draper EA et al (1991) The APACHE III prognostic system. Risk prediction of hospital mortality for critically ill hospitalized adults. Chest 100:1619-1636. https://doi.org/10.1378/chest.100.6.1619

4. Le Gall J-R, Lemeshow S, Saulnier F (1993) Simplified Acute Physiology Score (SAPS II) based on a European / North American multicenter study. JAMA 270:2957-2963. https://doi.org/10.1001/jama.270.24.2957

5. Metnitz PGH, Moreno RP, Almeida E et al (2005) SAPS 3 from evaluation of the patient to evaluation of the intensive care unit. Part 1: Objectives, methods and cohort description. Intensive Care Med 31:1336-1344. https://doi.org/10.1007/s00134-005-2762-6

6. Moreno RP, Metnitz PGH, Almeida E et al (2005) SAPS 3-from evaluation of the patient to evaluation of the intensive care unit. Part 2: development of a prognostic model for hospital mortality at ICU admission. Intensive Care Med 31:1345-1355. https://doi.org/10.1007/ s00134-005-2763-5

7. Harrison DA, Parry GJ, Carpenter JR et al (2007) A new risk prediction model for critical care: the Intensive Care National Audit \& Research Centre (ICNARC) model. Crit Care Med 35:1091-1098. https://doi.org/10. 1097/01.CCM.0000259468.24532.44

8. Brinkman S, Abu-Hanna A, de Jonge E, de Keizer NF (2013) Prediction of long-term mortality in ICU patients: model validation and assessing the effect of using in-hospital versus long-term mortality on benchmarking. Intensive Care Med 39(11):1925-1931. https://doi.org/10.1007/ s00134-013-3042-5
9. Kramer AA, Higgins TL, Zimmerman JE (2015) Comparing observed and predicted mortality among ICUs using different prognostic systems: why do performance assessments differ? Crit Care Med 43:261-269. https:// doi.org/10.1097/CCM.0000000000000694

10. Solomon PJ, Kasza J, Moran JL, Australian and New Zealand Intensive Care Society (ANZICS) Centre for Outcome and Resource Evaluation (CORE) (2014) Identifying unusual performance in Australian and New Zealand intensive care units from 2000 to 2010. BMC Med Res Methodol 14:53. https://doi.org/10.1186/1471-2288-14-53

11. Rothen HU, Stricker K, Einfalt J et al (2007) Variability in outcome and resource use in intensive care units. Intensive Care Med 33:1329-1336. https://doi.org/10.1007/s00134-007-0690-3

12. Soares M, Bozza FA, Angus DC et al (2015) Organizational characteristics, outcomes, and resource use in 78 Brazilian intensive care units: the ORCHESTRA study. Intensive Care Med 41:2149-2160. https://doi.org/10. 1007/s00134-015-4076-7

13. Reinikainen M, Mussalo P, Hovilehto S et al (2012) Association of automated data collection and data completeness with outcomes of intensive care. A new customised model for outcome prediction. Acta Anaesthesiol Scand 56:1114-1122. https://doi.org/10.1111/j.1399-6576. 2012.02669.X

14. Keene AR, Cullen DJ (1983) Therapeutic Intervention Scoring System: update 1983. Crit Care Med 11:1-3. https://doi.org/10.1097/00003246198301000-00001

15. OECD (2020) Conversion rates: purchasing power parities (PPP), OECD Data. OECD, Paris. https://doi.org/10.1787/1290ee5a-en

16. Lindemark F, Haaland $\varnothing A$, Kvåle R et al (2017) Costs and expected gain in lifetime health from intensive care versus general ward care of 30,712 individual patients: a distribution-weighted cost-effectiveness analysis. Crit Care 21:220. https://doi.org/10.1186/s13054-017-1792-0

17. Tan SS, Bakker J, Hoogendoorn ME et al (2012) Direct cost analysis of intensive care unit stay in four European countries: applying a standardized costing methodology. Value Health 15:81-86. https://doi.org/10. 1016/j.jval.2011.09.007

18. Edbrooke DL, Minelli C, Mills GH et al (2011) Implications of ICU triage decisions on patient mortality: a cost-effectiveness analysis. Crit Care 15:R56. https://doi.org/10.1186/cc10029

19. Sakr Y, Moreira CL, Rhodes A et al (2015) The impact of hospital and ICU organizational factors on outcome in critically ill patients: results from the Extended Prevalence of Infection in Intensive Care study. Crit Care Med 43:519-526. https://doi.org/10.1097/CCM.0000000000000754

20. Banerjee R, Naessens JM, Seferian EG et al (2011) Economic implications of nighttime attending intensivist coverage in a medical intensive care unit. Crit Care Med 39:1257-1262. https://doi.org/10.1097/CCM.0b013 e31820ee1df 